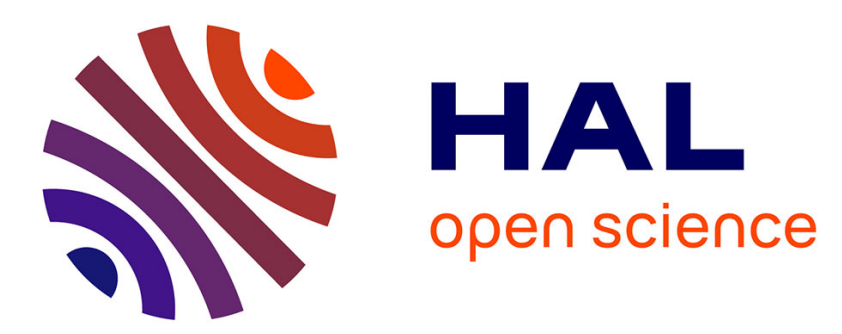

\title{
Étude du bruit ferromagnétique en fonction de la nature des blocages des parois de Bloch
}

\author{
A. Debus, A. Vannes
}

\section{To cite this version:}

A. Debus, A. Vannes. Étude du bruit ferromagnétique en fonction de la nature des blocages des parois de Bloch. Revue de Physique Appliquée, 1988, 23 (8), pp.1347-1353. 10.1051/rphysap:019880023080134700 . jpa-00245952

\section{HAL Id: jpa-00245952 https://hal.science/jpa-00245952}

Submitted on 1 Jan 1988

HAL is a multi-disciplinary open access archive for the deposit and dissemination of scientific research documents, whether they are published or not. The documents may come from teaching and research institutions in France or abroad, or from public or private research centers.
L'archive ouverte pluridisciplinaire HAL, est destinée au dépôt et à la diffusion de documents scientifiques de niveau recherche, publiés ou non, émanant des établissements d'enseignement et de recherche français ou étrangers, des laboratoires publics ou privés. 


\title{
REVUE DE PHYSIQUE APPLIQUÉE
}

\section{Étude du bruit ferromagnétique en fonction de la nature des blocages des parois de Bloch}

\author{
A. Debus et A. Vannes \\ G.E.P.P.M., U.A. 341, I.N.S.A., Bât. 303, 69621 Villeurbanne Cedex, France
}

(Reçu le 12 janvier 1988, révisé le 30 mars 1988, accepté le 22 avril 1988)

\begin{abstract}
Résumé. - Le bruit ferromagnétique est engendré principalement par le mouvement des parois de Bloch. Ces mouvements sont fonction des défauts de structure, notamment des dislocations et de leur arrangement. A l'aide d'un modèle fondé sur les fonctions de distribution dite de Gram-Charlier, nous montrons que le bruit est produit par deux sources indépendantes et que chacune d'elles est liée à un type d'interaction paroi-défaut.

Abstract. - The ferromagnetic noise is generated mainly by the movement of the Bloch's walls. These movements depend of the structure defects, such as dislocations and dislocation cells. Using a model based on the Gram-Charlier's distribution law, we show that the noise comes from two different origins and that each of them is bounded with the nature of the interaction defect-wall.
\end{abstract}

\section{Introduction.}

Soumettre un matériau ferromagnétique à un cycle d'hystérésis revient à provoquer le déplacement des parois de Bloch et la rotation des domaines de Weiss qui le constituent. Ces mouvements induisent des perturbations du champ magnétique externe qui sont captées à l'aide de bobines appropriées. La somme des impulsions intégrées sur une demipériode constitue le bruit ferromagnétique ou effet Barkhausen [1-3]. De nombreux auteurs ont montré que les déplacements des parois dépendent des interactions de celles-ci avec la microstructure, les dislocations, les précipités, ... [4-6].

La plupart des théories développées reposent sur l'hypothèse d'une distribution statistique de l'ensemble des blocages. Ainsi, il a pu être mis en évidence [1-3] que l'équation du mouvement d'une paroi, sous l'action d'un champ $H$ et d'une force $F(z)$, est donnée par la relation concernant une unité de surface de paroi à $180^{\circ}$ :

$$
M z^{\prime \prime}+\beta z^{\prime}-F(z)=2 I_{\mathrm{s}} H \cos \phi
$$

avec :

$M$ : masse effective de la paroi,

$z \quad$ : position de la paroi, $\beta$ : coefficient d'amortissement visqueux,

$I_{\mathrm{s}}$ : aimantation à saturation,

$\phi$ : angle formé par le champ appliqué et la direction de magnétisation.

La masse des parois étant très faible (de l'ordre de $10^{-10} \mathrm{~g} . \mathrm{cm}^{-2}$ ), on peut négliger le terme d'inertie $M_{z}$ ce qui permet d'écrire :

$$
Z^{\prime}=2 I_{\mathrm{s}}\left[H \cos \phi-H_{F}(z)\right] / \beta ;
$$

avec :

$$
H_{F}(z)=-\beta F(z) / 2 I_{\mathrm{s}} \text {. }
$$

$H_{F}(z)$ représente un champ retardateur s'appliquant sur la paroi. Il est décrit par une fonction complexe dépendant de tout l'environnement, donc de l'énergie à fournir à la paroi ou, plus exactement, à un ensemble de parois par effet d'avalanche [2] pour provoquer leurs déplacements.

Nous proposons, à partir d'une analyse expérimentale, de formaliser la fonction enveloppe de ce champ retardateur et de montrer que les mouvements des parois peuvent être classés selon deux familles principales, fonction des types d'interactions entre les parois et les défauts de structure. 


\section{Etude générale du bruit.}

2.1 DÉTECTION DU BRUIT. - Un appareillage spécifique a été conçu par le CETIM : [7]. Il permet :

- d'une part de créer un champ magnétique variant linéairement entre deux valeurs extrêmes de niveaux suffisamment élevés pour obtenir la saturation du matériau testé et ce à des fréquences comprises entre $0,05 \mathrm{~Hz}$ et quelques $\mathrm{Hertz}$; dans cette étude, nous avons retenu $0,1 \mathrm{~Hz}$;

- d'autre part une chaîne de mesure du bruit constitué :

- d'un capteur : celui-ci est composé soit d'une bobine en fil de cuivre, soit d'un ensemble de ferrite en forme de $U$, de $E$ ou de tige sur lesquels est enroulé un fil de cuivre constituant un bobinage ayant, selon le cas, entre 700 et 6200 spires avec un diamètre de 0,03 à $0,1 \mathrm{~mm}$. Une étude systématique de ces capteurs a été réalisée [8] ; elle nous permet de choisir celui qui est le mieux adapté aux caractéristiques spécifiques du bruit à percevoir ;

- d'une chaîne électronique et informatique de traitement des données.

Dans ces conditions, nous avons étudié du nickel pur (4 N) dans différents états : recuit, écroui, recristallisé ou sous contrainte. Les échantillons retenus sont des prismes de $2 \times 10 \times 100 \mathrm{~mm}^{3}$ de dimensions. La figure 1a représente l'enveloppe du bruit correspondant à un nickel recuit, la figure $1 \mathrm{~b}$ celle d'un nickel écroui à $35 \%$ par déformation homogène : on peut observer des modifications très sensibles entre les deux profils.

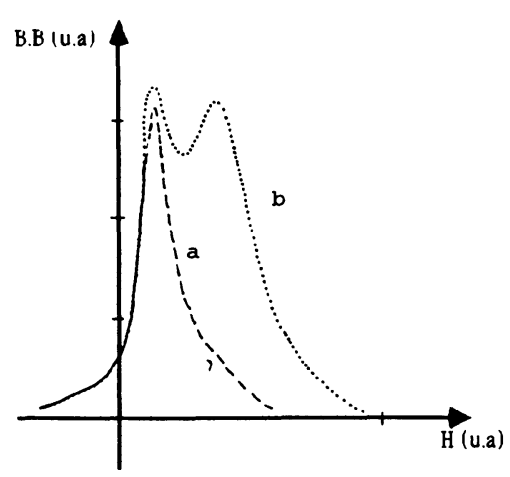

Fig. 1. - Profil expérimental du Bruit Barkhausen relatif au nickel : (a) nickel recuit ; (b) nickel écroui.

[Experimental shape of the Barkhausen's noise: (a) annealed nickel ; (b) cold worked nickel.]

2.2 INTERPRÉtATION. - Certains auteurs, dont Tourrenc et al. [9-10] ont émis l'hypothèse que le bruit est d'origine purement statistique et donc que la fonction $H(z)$ est une fonction aléatoire. Après diverses tentatives fondées sur cette hypothèse, nous avons été amenés, à partir des données expérimenta- les, à élargir le raisonnement et à considérer que les interactions dépendaient des dimensions des défauts. Tourrenc [12] a pu alors montrer, d'un point de vue strictement théorique, l'intérêt de cette approche et que le bruit pouvait résulter de deux types de sources ayant chacune son propre champ retardateur. Autrement dit, le modèle expérimental proposé est fondé sur les hypothèses suivantes:

- le bruit est d'origine purement statistique, mais les obstacles existant au sein du matériau engendrent deux types d'interactions n'ayant aucune synergie entre elles. En première approximation, comme cela sera montré ultérieurement, nous pouvons admettre qu'il s'agisse, d'une part, de défauts de dimensions inférieures à celles des parois de Bloch ayant pour effet de diminuer l'énergie interne des parois [1], et, d'autre part, de ceux de dimensions égales ou supérieures à celles-ci ayant pour effet la création, par exemple, de domaines de fermeture ;

- compte tenu de la structure expérimentale du bruit et de son caractère assymétrique, nous avons cherché une fonction statistique la plus générale possible capable de décrire chacune des sources et permettant un traitement analytique du problème : nous avons donc retenu la fonction dite de GramCharlier [11] qui s'écrit :

$$
\begin{aligned}
y= & \frac{A \mathrm{e}^{-x^{2} / 2}}{2 M \cdot(\mathrm{ET})} \times \\
& \times\left(1-\frac{G_{1}\left(X^{3}-3 X\right)}{6}+\frac{G_{2}\left(X^{4}-6 X^{2}+3\right)}{24}\right)
\end{aligned}
$$

avec :

$$
X=(x-M) / \mathrm{ET}
$$

avec :

ET : écart type,

$M \quad:$ moyenne,

$A \quad$ : aire,

$G_{1}=\mu_{3} / \mu_{2}^{3 / 2} \quad:$ coefficient d'assymétrie,

$G_{2}=\left(\mu_{4}^{-3} \mu_{2}^{2}\right) / \mu_{2}^{2} \quad:$ coefficient d'aplatissement, $\mu_{r}=\left(x-m_{1}\right)^{r} f(x) \mathrm{d} x$,

$m_{1} \quad$ : espérance mathématique, $f(x)$

: densité de probabilité.

Cette fonction prend en compte les moments d'ordres 3 et 4 , ce qui permet d'intégrer des écarts sensibles à la loi normale.

La méthode de convolution retenue consiste à rechercher les deux fonctions de Gram-Charlier qui permettent de reconstituer, par simple addition [8], le bruit enregistré. Il est possible de résoudre ce problème à l'aide d'une méthode itérative qui repose sur la mise en œuvre de l'algorithme de NewtonRaphson fondé sur la minimisation d'un indicateur des moindre carrés calculé à partir de la résolution d'un système matriciel qui, dans la quasi-totalité des cas, aboutit à une solution convergente. Cette 
méthode conduit à une solution déterminée par une minimisation d'un paramètre d'ajustement (ou paramètre d'erreur) calculé à partir de la courbe expérimentale et la somme des courbes ajustées ; pour un niveau d'erreur fixé, la solution obtenue est unique. La figure 2 présente une série de résultats qui montrent la qualité des ajustements obtenus; on peut noter que, dans les conditions de la représentation, les écarts entre les courbes expérimentales et les courbes ajustées ne sont pas apparents sur le tracé.
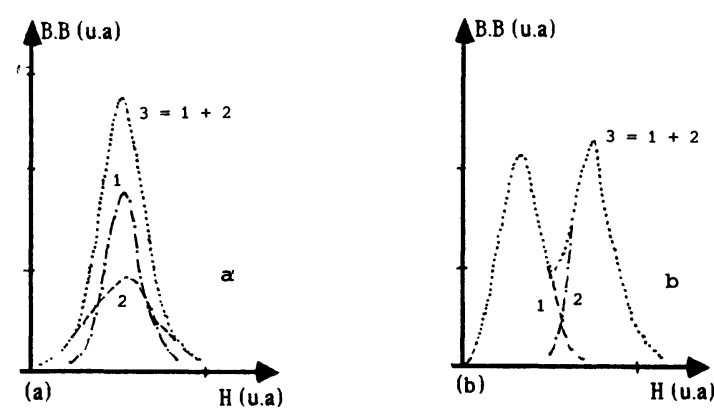

Fig. 2. - Décomposition du profil Barkhausen par la méthode de Newton-Raphson: (a) nickel recuit; (b) nickel fortement écroui.

[Decomposition of the Barkhausen's shape using a Newton-Raphson method : (a) annealed nickel ; (b) high cold worked nickel.]

Chaque courbe peut alors être qualifiée par les différents paramètres représentatifs d'une fonction statistique et, notamment :

- la moyenne $M$ ou la position du maximum $H_{\mathrm{M}}$. Les valeurs du champ ainsi définies sont voisines du champ coercitif, l'écart entre ces deux paramètres est fonction de l'assymétrie de la courbe et donc de l'homogénéité de la population d'ancrage considérée ; ils peuvent être notamment corrélés aux contraintes macroscopiques (appliquées ou résiduelles) au même titre que le champ coercitif ;

- l'amplitude $\left(A_{\mathrm{M}}\right)$ et l'aire sous courbe $(S)$ sont des paramètres sensibles à la microstructure, ils sont proportionnels au nombre de décrochements $(n)$ et à la taille des volumes de corrélation $\left(V_{\mathrm{c}}\right)$;

- l'écart type (ET), ainsi que les coefficients d'aplatissement et d'assymétrie, donnent une information directe sur l'homogénéité des ancrages et ils semblent dépendre des contraintes internes de deuxième et troisième ordre qui sont celles qui résultent des accommodations élastiques engendrées par les incompatibilités de déformations entre grains ou au voisinage des défauts de structures.

L'étude expérimentale, qui va suivre, va permettre de préciser le contenu effectif des différents paramètres.

\section{Etude du nickel.}

3.1 INFluenCE DES TAILleS DE GRAINS. - Par un maintien au four à $850^{\circ} \mathrm{C}$ pendant $2,4,5,10,20$ et $180 \mathrm{mn}$ d'éprouvettes de nickel préalablement écrouies à $35 \%$, il a été possible de faire varier la taille de grain entre les indices normalisés $G=4$ et $G=2$ (c'est-à-dire un diamètre moyen compris entre 0,00885 et $0,117 \mathrm{~mm}$ ). La figure 3 présente un exemple de décomposition du signal enregistré après un traitement thermique de $10 \mathrm{mn}$.

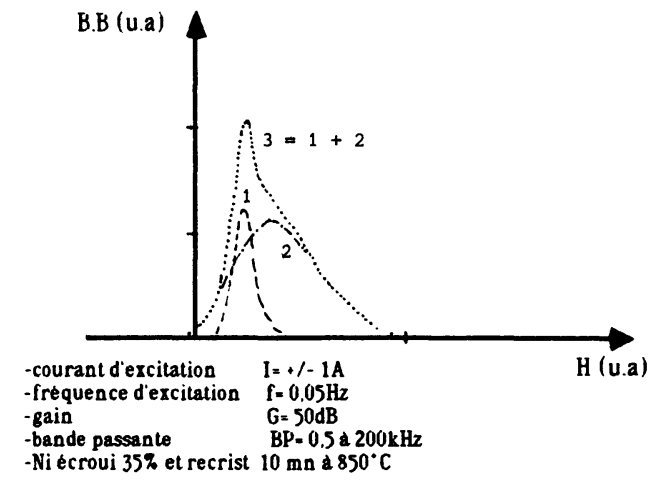

Fig. 3. - Profil expérimental du bruit ferromagnétique pour le nickel recristallisé.

[Decomposition of the Barkhausen's shape in the recristallized nickel.]

La figure 4 rapporte la variation d'amplitude des pics 1 et 2 obtenus par la décomposition du signal global. Seul le pic 1 évolue dans ce cas de figure ; cette évolution se traduit par une chute de l'amplitude de ce pic dès les premières minutes du traitement. Les autres paramètres $\left(\mathrm{ET}, H_{\mathrm{M}}, M, \ldots\right)$ n'évoluent pas de manière significative.

3.2 INFLUENCE DES DÉFAUTS LINÉAIRES TYPE DISLOCATION. - Les déformations imposées aux éprouvettes d'essai ont été réalisées par traction homogène. La figure 5 représente la courbe de traction du nickel en coordonnées rationnelles $\left(\sigma_{\mathrm{r}}, \varepsilon_{\mathrm{v}}\right)$. La figure 6 décrit les variations de $A$, et la figure 7 celles de $M$ pour chacun des pics (1 et 2 ) en fonction de la déformation vraie. L'ensemble de ces résultats peut être exprimé par les relations suivantes :

- amplitude du maximum $A_{\mathrm{M}_{0}}$;

(*) pic 1 : quasi constant à l'exception de la phase initiale qui peut être attribuée à des effets de déplacements irréversibles de certaines parois [13] ;

(*) pic 2 :

$$
A_{\mathrm{M}_{2}}=1,7 \varepsilon_{\mathrm{v}}+A_{\mathrm{M}_{0}}
$$

$\operatorname{avec} A_{\mathrm{M}_{0}}=0,4$; 


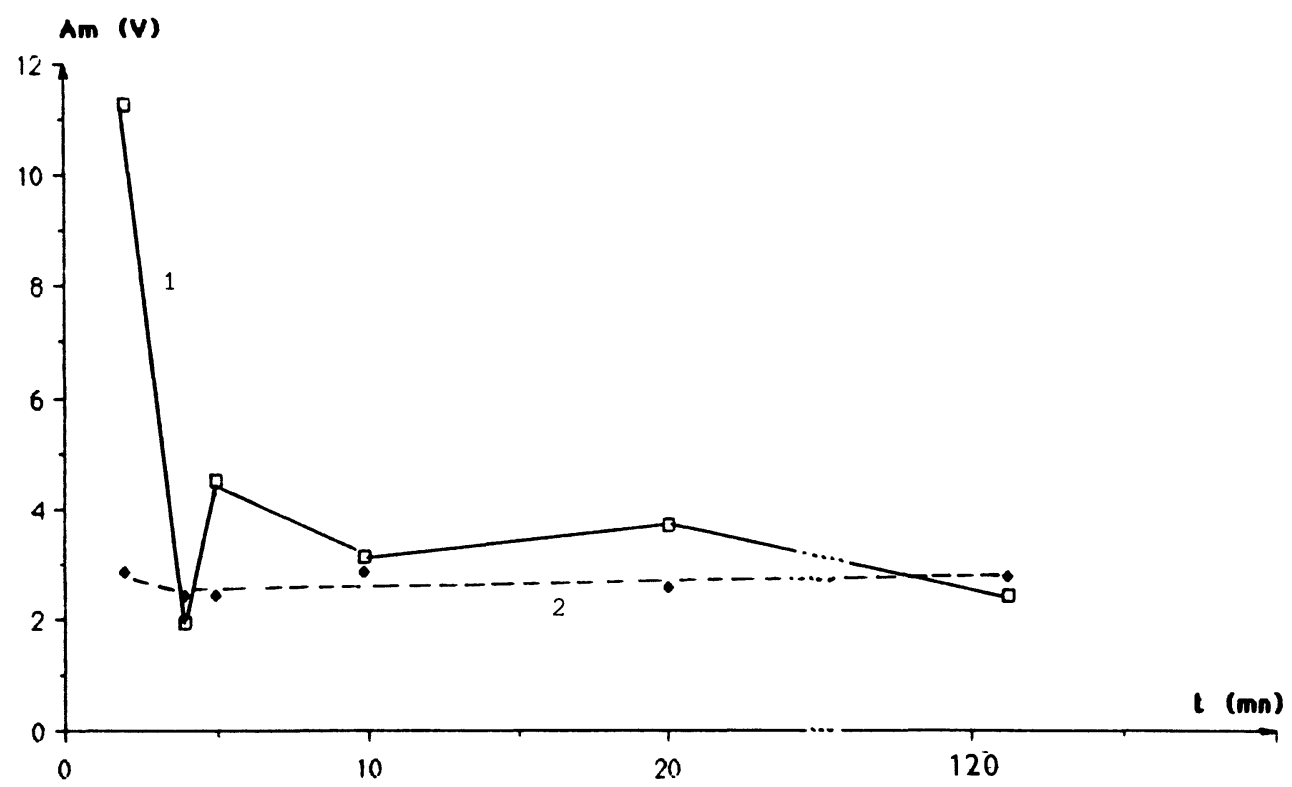

Fig. 4. - Evolution des amplitudes maximales du bruit $A_{\mathrm{M}}(1$ et 2$)$ en fonction du temps de maintien $t$ à $850^{\circ} \mathrm{C}$. [Evolution of the maximum amplitude $(A)$ of the noise in relation to the maintained time $t$ at $850^{\circ} \mathrm{C}$.]

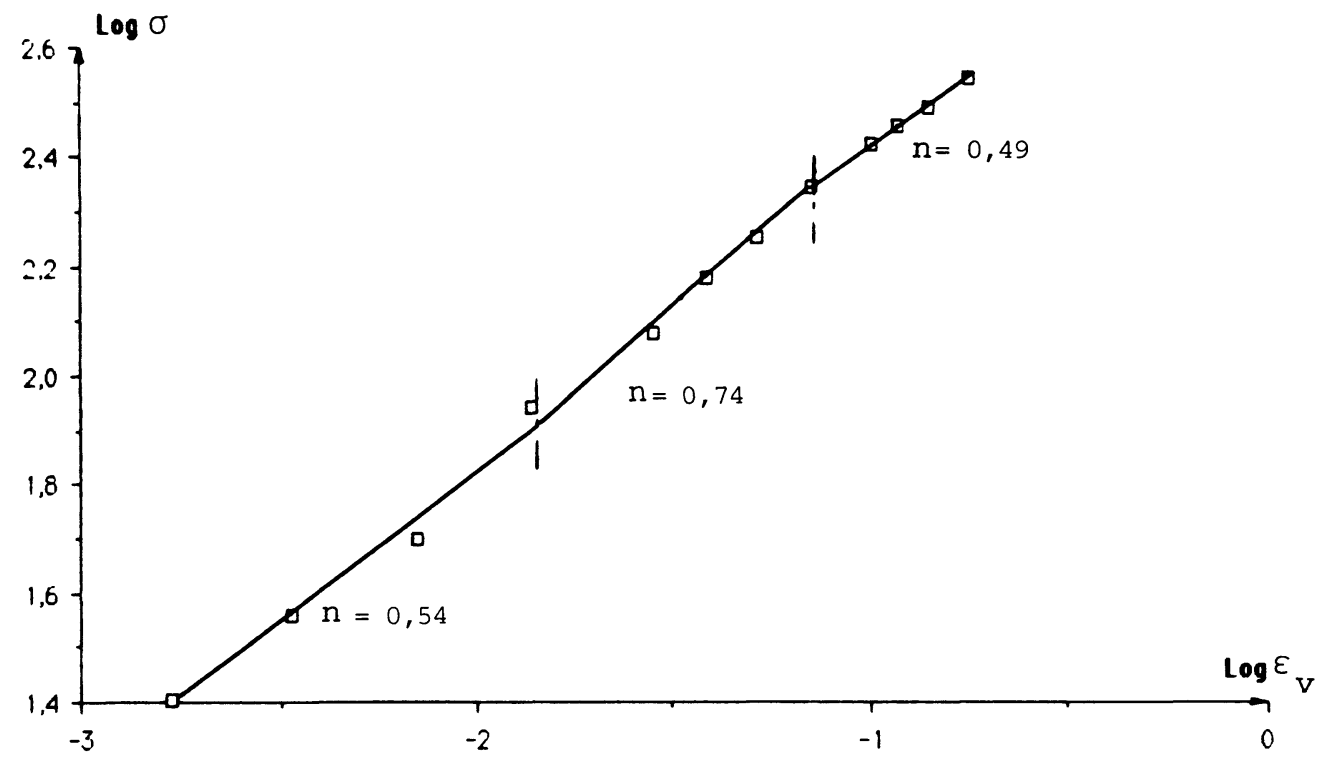

Fig. 5. - Courbe de traction rationnelle exprimée en coordonnées de Ludwick.

[Strain-stress curves of the nickel in Ludwick's coordinates.]

- champ retardateur $H_{F}$ (ou moyenne) ;

$\left.{ }^{*}\right)$ pic 1 :

$$
\Delta H_{F_{1}}=H_{F_{1\left(\varepsilon_{\mathrm{v}}\right)}}-H_{F_{1\left(\varepsilon_{\mathrm{v}}=0\right)}}=0,7 \varepsilon_{\mathrm{v}}^{0,6} .
$$

Cette loi est identique à celle qui décrit les accommodations élastiques (contraintes internes de deuxième ordre $\sigma^{2}$ ) apparaissant lors des déformations plastiques, à savoir :

$$
\Delta \sigma_{i}^{2}=A \varepsilon_{\mathrm{v}}^{n} ;
$$

avec $n=$ taux de consolidation [14].

(*) pic 2 :

Deux cas sont à considérer :

$$
\begin{array}{ll}
\varepsilon_{\mathrm{v}} \leqslant 20 \% & \Delta H_{F}=0,76 \varepsilon_{\mathrm{v}}+0,24 ; \\
\varepsilon_{\mathrm{v}}>20 \% & \Delta H_{F}=0,37 \varepsilon_{\mathrm{v}}+0,3 .
\end{array}
$$

Ce pic se caractérise en outre par un écart type important. Il est à noter que le taux de transition de $20 \%$ correspond au passage d'un arrangement des 


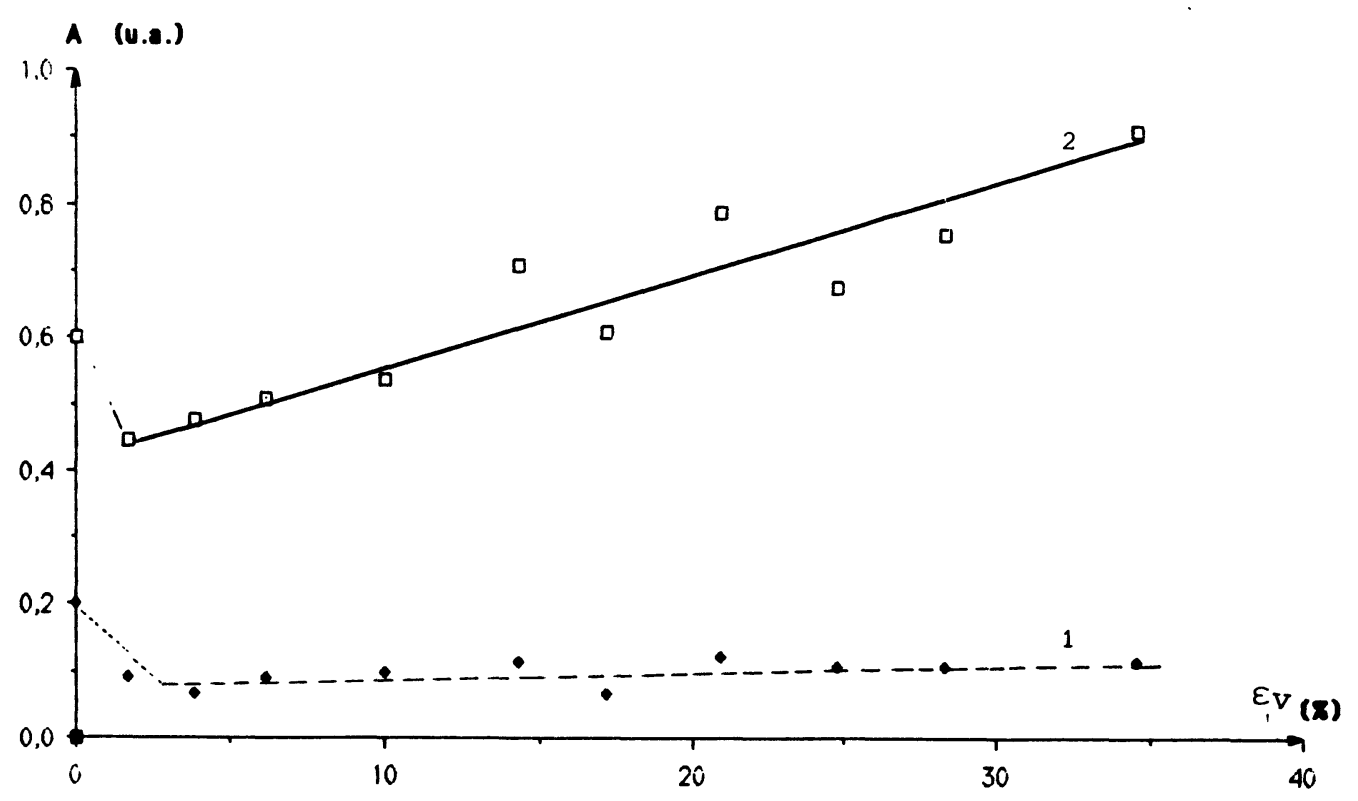

Fig. 6. - Evolution de l'amplitude maximale $A$ en fonction de la déformation rationnelle $\varepsilon$.

[Evolution of the maximum amplitude $(A)$ in relation to the true strain.]

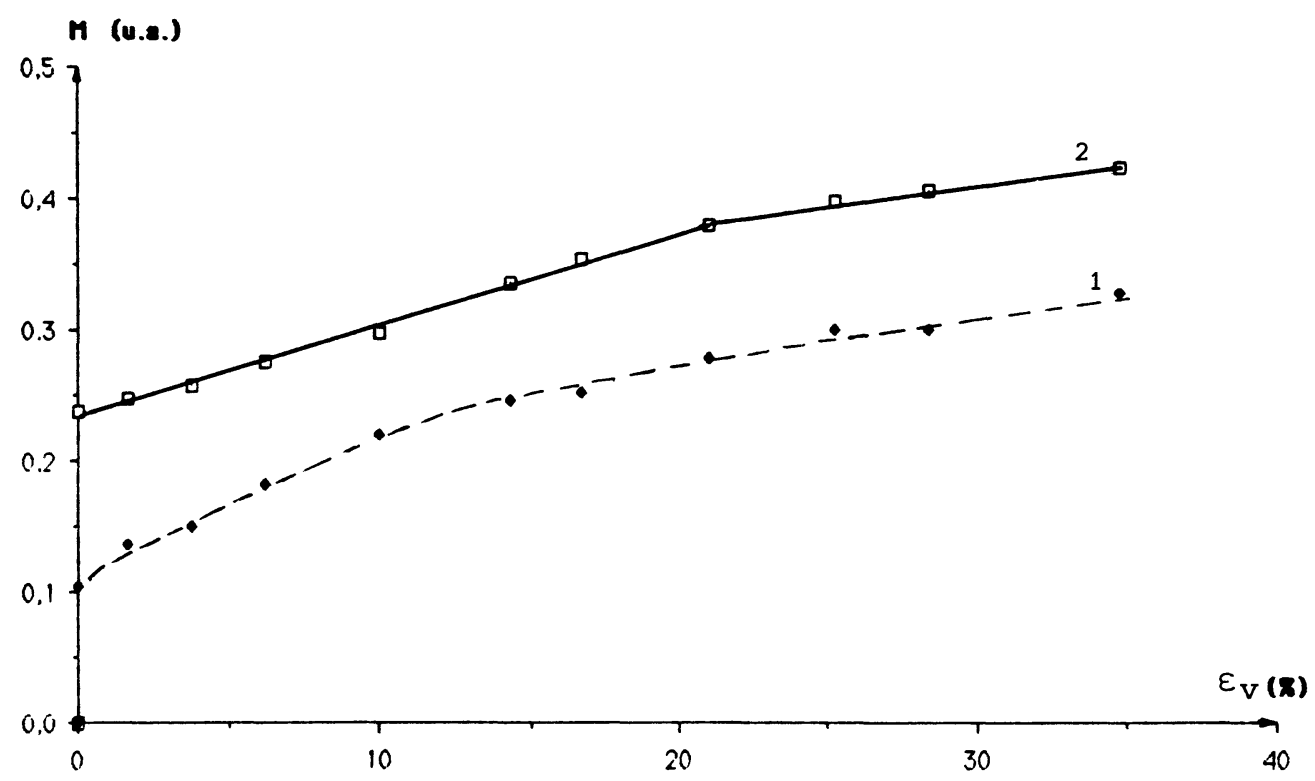

Fig. 7. - Evolution de la position de la moyenne en fonction de la coordonnée rationnelle $\varepsilon$.

[Evolution of the maximum position in relation to the true strain.]

dislocations correspondant à un enchevêtrement à celui correspondant à une répartition cellulaire [8].

Il ressort de cette analyse que c'est le pic 2 qui décrit les interactions parois-défauts, l'évolution du pic 1 résultant d'un effet indirect par la création de contraintes internes.

\subsection{ETUDE DES CONTRAINTES MACROSCOPI-} QUES. - Des essais de flexion quatre points sont réalisés à l'aide d'une machine spécialement conçue à cet effet; il s'agit d'une micromachine à bruit Barkhausen intégré [8]. Ils permettent de mettre en évidence le effets d'une traction ou d'une compression tout en restant dans le domaine élastique et en se plaçant dans des conditions comparables.

Les évolutions des différents paramètres relatifs à chacun des pics sont linéaires et résumées dans le tableau I.

L'application d'une contrainte provoque une modification de la cartographie des parois en produi- 
Tableau I.

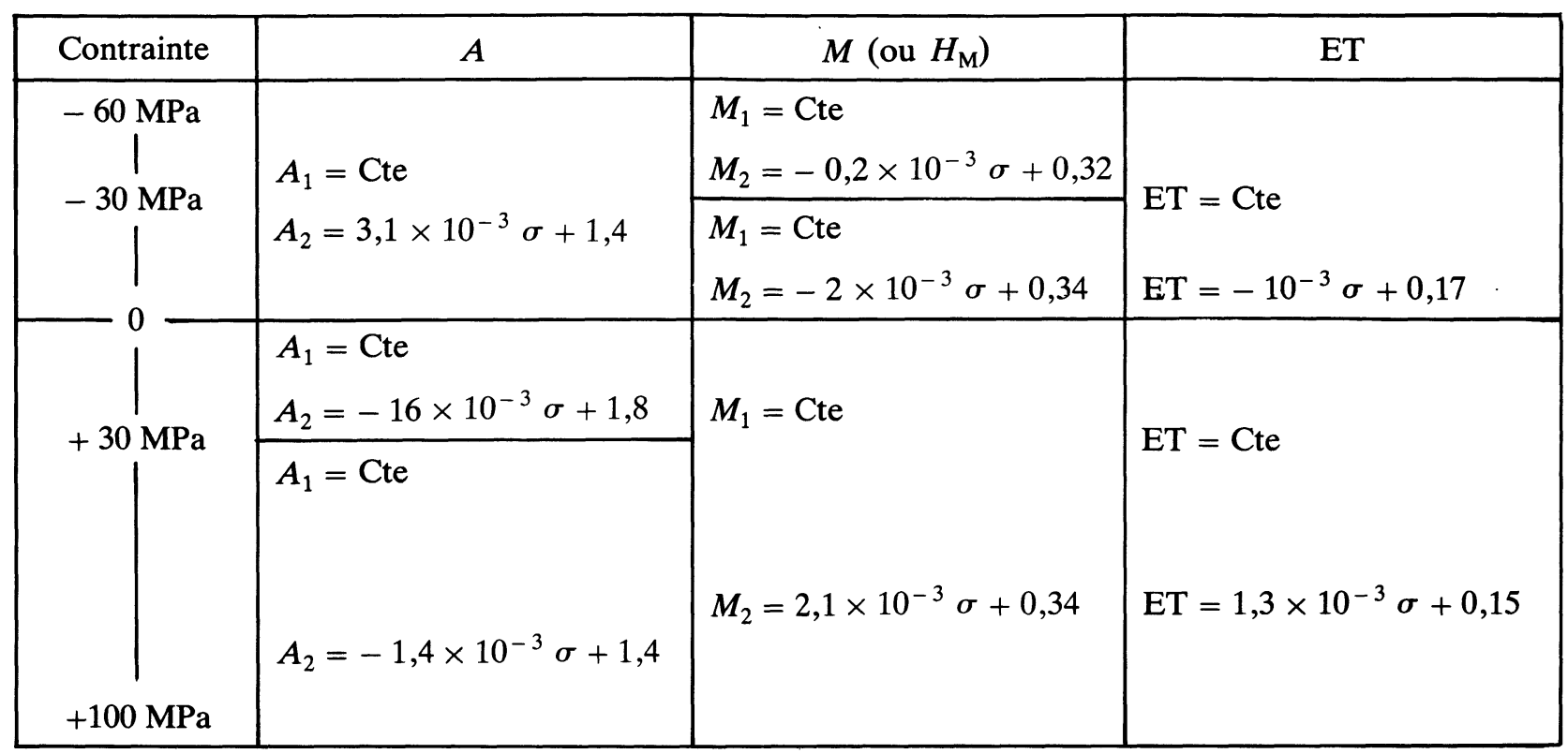

sant un mouvement des parois à $71^{\circ}$ et $109^{\circ}$ dites abusivement à $90^{\circ}$ [15]. Dans le cas des matériaux magnétostrictifs négatifs (cas du nickel), l'application d'une contrainte de compression augmente le nombre de parois à $180^{\circ}$ génératrices du bruit d'origine magnétique - c'est l'inverse pour une contrainte de traction - ce qui provoque un accroissement de l'amplitude du pic (et de l'aire) [6] : ces variations sont linéaires et n'affectent que le pic 2 .

En première approximation, on peut admettre que l'effet magnétomécanique est équivalent à l'application d'un champ fictif $H_{\mathrm{f}}$, positif dans le cas du nickel pour une contrainte de compression $[16$, 17], ce qui conduit à diminuer l'énergie à fournir aux parois pour les mettre en mouvement (c'est l'inverse pour une traction). En effet, sous l'influence d'une contrainte, la cartographie générale des parois est modifiée par des déplacements des parois à $71^{\circ}$ et à $109^{\circ}$ ce qui entraîne, par voie de conséquence, la modification des paramètres du bruit. Ce champ peut s'écrire :

$$
H_{\mathrm{f}}=\frac{3}{2} \lambda_{\mathrm{s}} / I_{\mathrm{s}} \sigma=k \sigma
$$

$I_{\mathrm{s}}:$ module du vecteur aimantation à saturation, $\lambda_{\mathrm{s}}$ : coefficient de magnétostriction à saturation.

Ce champ $H_{\mathrm{f}}$ est décrit par les évolutions de la moyenne (ou de la position du maximum) du pic 2 . Les variations d'amplitude et d'écart type ne sont qu'une conséquence indirecte des effets des contraintes macroscopiques sur la population des parois.

3.4 INTERPRÉTATION. - Les trois cas de figures étudiés permettent d'identifier le type d'interaction responsable de l'apparition de chacun des pics mis en évidence par la décomposition du signal global.

Le pic 1, associé à un champ retardateur d'intensité moyenne faible, semble décrire des interactions entre défauts ayant des dimensions comparables à celles des parois du type joint de grain. Il est sensible aux effets de contraintes internes de deuxième ordre, c'est-à-dire aux accommodations élastiques entre grains résultant des hétérogénéités de déformation.

Le pic 2, associé à un champ retardateur moyen plus élevé que le précédent, semble rendre compte des interactions parois-défauts ayant des dimensions inférieures à celles des parois du type dislocation, par exemple. Ces défauts entraînent une diminution de l'énergie moyenne des parois, ce qui conduit à des ancrages efficaces de celles-ci. Il en résulte un accroissement de l'énergie à fournir pour provoquer le mouvement de ces parois, cet accroissement et son évolution relative dépendent des arrangements des défauts. C'est également ce pic qui est sensible à l'effet d'une contrainte macroscopique.

\section{Conclusions.}

Cette étude montre que le bruit ferromagnétique est produit par deux sources qui tirent leur origine dans la nature des ancrages entre les parois de Bloch et les défauts de structure.

En première approximation, il est possible de classer ces défauts en deux familles :

- celle correspondant à des dimensions au moins égales à celles des parois qui introduit des champs retardateurs peu dispersés d'intensité relativement faible ; 
- celle correspondant à des dimensions inférieures à celles des parois qui introduit des champs retardateurs relativement dispersés - écart type des distributions important - et d'intensité moyenne relativement élevée.

Chacune des distributions peut être décrite par une fonction de Gram-Charlier qui est l'expression de l'enveloppe du champ retardateur s'appliquant aux parois.

La décomposition du signal en deux composantes permet de suivre aussi bien les effets d'un écrouissage, d'un traitement de recristallisation que l'influence d'une contrainte avec précision en identifiant chacun des paramètres sensibles.

\section{Bibliographie}

[1] Tirtro, S., Acta Polytech. Scand., Appl. Phys. 113 (1977) 1-80.

[2] Matzkanin, G. A., Beissner and Teller, C. E., Nondestr. Test. Inf. Anal. Cent. 79-2 (1979) 1-59.

[3] Pasley, R. L., Mater. Eval. (juillet 1970) pp. 157161.

[4] Karjalainen, L. P. and Mollaven, M., NDT Int. (avril 1979) pp. 51-55.

[5] Mihovski, N. M., Vicher, B. I. and Penndhey, H. G., Proc. 8th World conf. of nondestr. test. (Cannes) 1976, p. 1-4.

[6] Ranjan, R., BuCK, O. and ThOMPSON, R. B., J. Appl. Phys. 61 (1987) p. 3196-3198.

[7] Flambard, C., Spécifications techniques CETIMSenlis, Etude 45 H 377 (août 1981) p. 83.

[8] Debus, A., Thèse de doctorat $n^{\circ} 86$ ISAL 0026 (janvier 1987) p. 186.

[9] Flambard, C., Grossiord, J. L. et Tourrenc, P., CETIM-Senlis, Etude 15 H 377, rapport partiel $\mathrm{n}^{\circ} 1$ (avril 1979) p. 226.
[10] Bonnet, G., J. Phys. France 28 (1967) 913-930.

[11] Flambard, C., Grossiord, J. L. et Tourrenc, P., CETIM-Senlis, Etude $15 \mathrm{H} 377$, rapport partiel $\mathrm{n}^{\circ} 7$ (mars 1983) p. 25.

[12] Tinter, G., Mathématiques et statistiques par les économistes (Ed. Dunod, Paris) 1 (1965) 488.

[13] Herpin, A., Théorie du magnétisme INSTN-PUF, Paris (1968) p. 683.

[14] SIKEH, A., Imperfections in crystals, Interscience (1962) p. 213.

[15] Cousinou, E., AugustiniaK, B., Flambard, C. et Vannes, A. B., Mém. Sci. Rev. Métall. 5 (1985) 255-264.

[16] Degauque, J., Thèse de docteur ès-Sciences, $\mathrm{n}^{\circ} 745$, Université Paul Sabatier, Toulouse (1977) p. 159.

[17] Degauque, J., Astie, B., Porteseil, J. L. and Vergne, R., J. Magn. Magn. Mater. 26 (1982) 261-263. 